\title{
XPS Analysis of AuGeNi/Cleaved GaAs(110) Interface
}

\author{
Constantin-Catalin Negrila, Mihail Florin Lazarescu, Constantin Logofatu, \\ Costel Cotirlan, Rodica V. Ghita, Florica Frumosu, and Lucian Trupina
}

National Institute of Materials Physics, P.O. Box MG-7, Magurele, Bucharest, Romania

Correspondence should be addressed to Mihail Florin Lazarescu; mlazare49@yahoo.com

Received 22 April 2016; Revised 25 July 2016; Accepted 4 August 2016

Academic Editor: Bhanu P. S. Chauhan

Copyright (C) 2016 Constantin-Catalin Negrila et al. This is an open access article distributed under the Creative Commons Attribution License, which permits unrestricted use, distribution, and reproduction in any medium, provided the original work is properly cited.

\begin{abstract}
The depth composition of the thin layer alloy, AuGeNi, devoted to acting as an ohmic contact on n-GaAs(110) has been investigated by in situ XPS combined with Argon ion sputtering techniques. The fresh cleaved surfaces, supposed to be free of oxygen, were usually deposited with a $200 \mathrm{~nm}$ metallic layer in high vacuum conditions (better than $10^{-7}$ torr), by thermal evaporation, and annealed at a $430-450^{\circ}$ Celsius temperature for 5 minutes. About 18 sessions of ion Ar surfaces etching and intermediate XPS measurements were performed in order to reveal the border of the metal/semiconductor interface. The atomic concentrations of the chemical elements have been approximated. Au4f, Ga3d, Ga2p, As3d, As2p, Ni2p ${ }^{3 / 2}$, Ge3d, O1s, and C1s spectral lines were recorded. The $\mathrm{Au}, \mathrm{Ge}$, and $\mathrm{Ni}$ have a homogenous distribution while $\mathrm{Ga}$ and As tend to diffuse to the surface. Oxygen is present in the first layers of the surface while carbon completely disappears after the second etching step. The existence of an Au-Ga alloy was detected and XPS spectra show only metal Ni and Ge within the layer and at the interface. We tried to perform a study about the depth chemical composition profile analysis of AuGeNi layer on cleaved n-GaAs(110) by X-Ray Photoelectron Spectroscopy (XPS) technique.
\end{abstract}

\section{Introduction}

Gallium arsenide has been for a long time considered as one of the most important semiconductor materials besides silicon due to its attractive intrinsic electrical properties: direct energy and wider bandgap, higher carrier mobility, and high power and operating temperature [1]. A key element in GaAs devices technology consists in obtaining high quality ohmic contacts because the surface of this compound is often covered with a chemically unstable oxide layer. Developed on a trial-and-error basis, the AuGeNi alloyed ohmic contacts remain the most widely used for $\mathrm{n}$-GaAs devices manufacturing. This metallization system is characterized by low contact resistance, good thermal stability both during device fabrication and device operation, strong adhesion, and low metal sheet resistance [2].

In this system $\mathrm{Au}$ promotes $\mathrm{Ga}$ vacancies in $\mathrm{n}-\mathrm{GaAs}$ due to high solubility of $\mathrm{Ga}$ in $\mathrm{Au}$. Ge acts as a dopant element, diffusing into the lattice sites vacated by Ga, creating in this way a heavily doped n-type intermediate semiconductor layer that allows the tunneling mechanism which leads to the ohmic behavior. $\mathrm{Ni}$ acts as a catalyst during the alloying procedure to improve the uniformity, thermal and mechanical stability, and reduction of surface roughness [3].

The quality of the contact is affected however by diffusion and chemical reactions that take place during alloying, by extensive interactions between chemical components and $\mathrm{n}$-GaAs substrate. There have been tremendous efforts for correlating between the electrical properties and microstructures of AuGeNi ohmic contact materials. Extensive studies by X-Ray Diffraction (XRD), Secondary Ion Mass Spectroscopy (SIMS), Auger Electron Spectroscopy (AES), and Transmission Electron Spectroscopy (TEM) techniques combined with the electrical properties characterization have been carried out in the past [4-6].

Until now, there are no studies about the depth profile chemical composition of AuGeNi layer and the AuGeNi/ GaAs interface by X-Ray Photoelectron Spectroscopy (XPS).

Therefore, our research effort was to provide an extensive analysis for AuGeNi contacts on GaAs by using XPS combined with Ar+ sputtering to obtain depth profile 
information for the metallic layer and for the AuGeNi/GaAs interface. The main goal is to determine the chemical composition and interdiffusion processes in AuGeNi film and nGaAs at the interface.

\section{Experimental}

Samples were prepared by thermal evaporation of AuGeNi alloy ( $\mathrm{Au} 84 \%-\mathrm{Ge} 12 \%-\mathrm{Ni} 4 \%$ ) from filament on a $\mathrm{n}$ GaAs(110) cleaved surface. The $84 \% \mathrm{Au}, 12 \% \mathrm{Ge}$, and $4 \% \mathrm{Ni}$ mass proportions translate to the nearest $65 \% \mathrm{Au}, 25 \% \mathrm{Ge}$, and $10 \% \mathrm{Ni}$ atomic concentrations. This conversion is useful since the XPS technique measures atomic concentrations. The thickness of the n-GaAs wafer and thus the width of the deposited surface were $1 \mathrm{~mm}$. The $\mathrm{n}-\mathrm{GaAs}$ surface has been cleaved prior to metal evaporation in a high vacuum chamber maintained at $10^{-7}$ mbar. The deposition has been carried out in the same conditions, on the cleaved edge of the wafer. The thickness of the metallic layer was estimated at $200 \mathrm{~nm}$, an usual value for $\mathrm{n}-\mathrm{GaAs}$ electronic devices. A thermal annealing followed; the sample has been heated at $430^{\circ} \mathrm{C}$ for 5 minutes in a conventional furnace maintained at $10^{-2} \mathrm{mbar}$ pressure. The chemical composition of AuGeNi layer and metal/semiconductor interface was examined by XPS measurements in a high vacuum Specs installation. The $\mathrm{X}$-ray source was a monochromatic XR50M source operated at $300 \mathrm{~W}, 15 \mathrm{kV}$ with a radiation energy of $1486.6 \mathrm{eV}$ (Al $\mathrm{K}_{\alpha}$ ) and a FWHM (Full Width at Half Maximum) of $0.3 \mathrm{eV}$. The X-ray spot is smaller than the analyzed surface. The spectrometer is based on a PHOIBOS 150 hemispherical analyzer with an ultimate resolution of $0.44 \mathrm{eV}$ (defined as FWHM of recorded Ag3 $\mathrm{d}^{5 / 2}$ spectral line). The C1s, O1s, Ga3d, Ga2p, As3d, As2p, Au4f, Ge3d, and Ni2p spectra have been recorded with a Pass Energy of $20 \mathrm{eV}$ and a step of $0.05 \mathrm{eV}$. Each XPS measurement session has been followed by a 5-minute Ar+ etching stage, using an IQE11/35 ion gun operated at $3 \mathrm{kV}$ accelerating voltage. The etching rate was estimated at $15 \AA /$ minute. A total of 18 etching steps have been carried out. Spectra were processed using Spectral Data Processor v.2.3 (SDP) software. The spectra fitting was made using Voight functions and Shirley background subtraction method. For quantitative analyses, the sensitivity factors provided by the spectrometer manufacturer have been used.

\section{Results}

The chemical composition of the metallic layer has been determined using solely XPS technique. The surface has been analyzed before Ar etching and adventitious carbon and oxygen have been found, besides smaller quantities of Au and Ge. The Ga and As are present at the surface, the concentration of Ga being unexpectedly high. This is explainable, however, due to the long time of thermal annealing. This favors the $\mathrm{Ga}$ diffusion at the surface. In the first layers, $\mathrm{Ga}$ is almost completely oxidized. Large amounts of oxide were found in the case of As and Ge also. However, the metallic components of these two elements are much larger than that of Ga. Gold

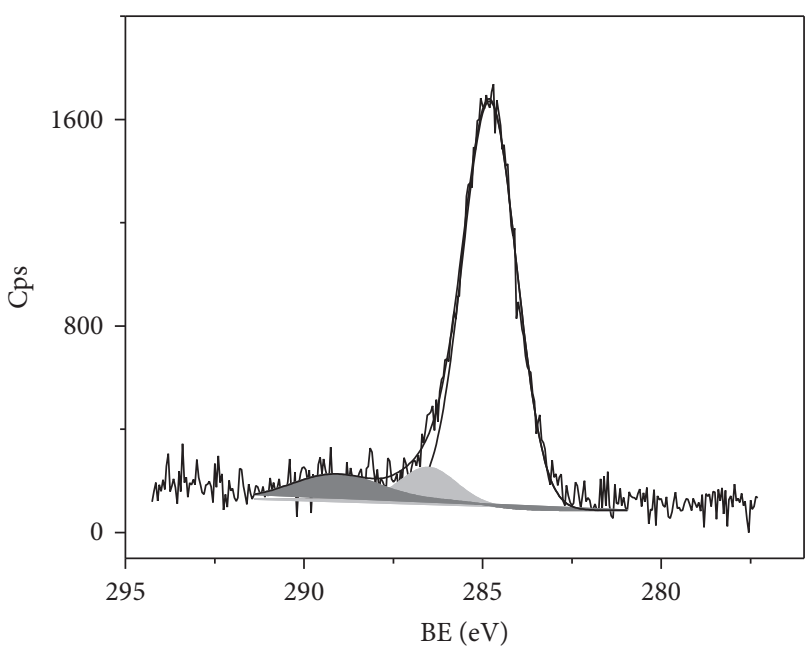

FIGURE 1: XPS spectra of C1s before etching.

is found as metal while Ni does not diffuse in the superficial layers of the sample.

The sample exhibits large quantities of adventitious carbon and oxygen at the surface with atomic concentrations of $22.4 \%$ and $34 \%$, respectively. The XPS spectrum of $\mathrm{Cls}$ line can be fitted with three Gaussian curves (Figure 1): one corresponding to $\mathrm{C}-\mathrm{C}$ and $\mathrm{C}-\mathrm{H}$ bonding located at $284.8 \mathrm{eV}$, another one located at higher binding energy $(286.6 \mathrm{eV})$ corresponding to $\mathrm{C}-\mathrm{O}$ bonding [7], and the last with a binding energy of $289.1 \mathrm{eV}$ corresponding to more complex bonds like $\mathrm{O}-\mathrm{C}-\mathrm{O}$ and $\mathrm{C}=\mathrm{O}$. After the first two ion etchings it is obvious that $\mathrm{C}$ does no longer exist at the surface.

From this point it is difficult to assess the correct charge neutralization, since $\mathrm{Au}$ might show a chemical shift, due to the presence of $\mathrm{Ga}$. In this respect, ensuring a good electrical contact between the sample and the ground of the spectrometer and the charge neutralization with a flood gun with a very low energetic electron beam of only $0.1 \mathrm{eV}$ were found particularly useful.

The O1s spectrum (Figure 2) is very well fitted with four components centered at $533 \mathrm{eV}, 531.05 \mathrm{eV}, 531.6 \mathrm{eV}$, and $531 \mathrm{eV}$.

The component located at $533 \mathrm{eV}$ is usually associated with adsorbed water [8] while the other three designate metallic oxides: the peak located at $531 \mathrm{eV}$ is attributed to $\mathrm{Ga}_{2} \mathrm{O}_{3}$ [9], that located at $531.6 \mathrm{eV}$ to $\mathrm{As}_{2} \mathrm{O}_{3}$ [10], and that at $532 \mathrm{eV}$ to $\mathrm{GeO}_{2}$ [11]. The peak located at $531.6 \mathrm{eV}$ may be also associated with $\mathrm{C}-\mathrm{O}$ and $-\mathrm{OH}$ bonding [12].

After the first sputtering session, only the peak associated with gallium oxide remains visible and oxygen starts to decrease gradually. The metal-semiconductor interface is found to be revealed after approximately 17 sputtering sessions. The bulk metallic layer displays as a mixture of Ga, $\mathrm{As}, \mathrm{Au}, \mathrm{Ge}$, and Ni. Figure 3 presents the atomic relative concentrations of $\mathrm{Au}, \mathrm{Ge}$, and $\mathrm{Ni}$. It can be seen that all three components have constant behavior, one relative to the others, and that the homogeneity has been achieved.

After etching step number 15, the metal/GaAs interface started to reveal. The concentrations of $\mathrm{Au}$ and $\mathrm{Ge}$ begin to 


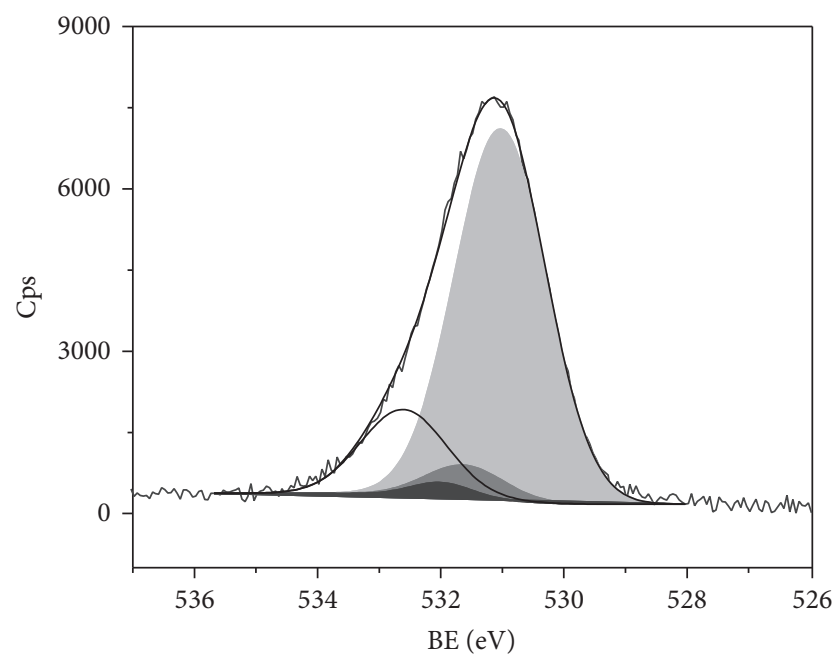

FIGURE 2: XPS spectra of O1s before etching.

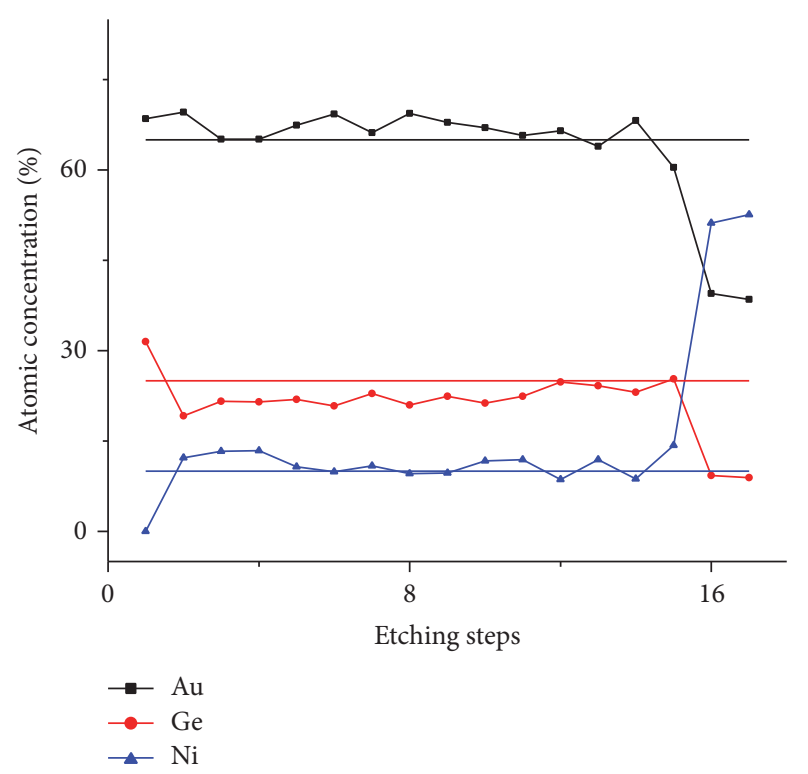

FIGURE 3: Relative atomic concentrations of $\mathrm{Au}, \mathrm{Ge}$, and $\mathrm{Ni}$ within the layer; solid lines are theoretical concentrations.

decline sharply compared with that of $\mathrm{Ni}$. The presence of $\mathrm{Ni}$ at the interface is desirable since $\mathrm{Ni}$ acts as a wetting agent. Its diffusion in GaAs produces a reduction in its Gibbs potential which in turn leads to an enhanced diffusion of Ge in GaAs. $\mathrm{Ni}$ also enhances the homogeneity and uniform alloying of the metallic layer.

Figure 4 presents the depth profile of AuGeNi layer and the interface with the n-GaAs semiconductor. From this figure it can be seen that while the As concentration tends to grow steadily, the Ga concentration varies in a different manner: it is greater in the first layers; then it starts to decrease to a minimum of $35 \%$ and then is growing once more. This last growing is explained through the decrease of $\mathrm{Au}$ concentration which is more aggressively removed from

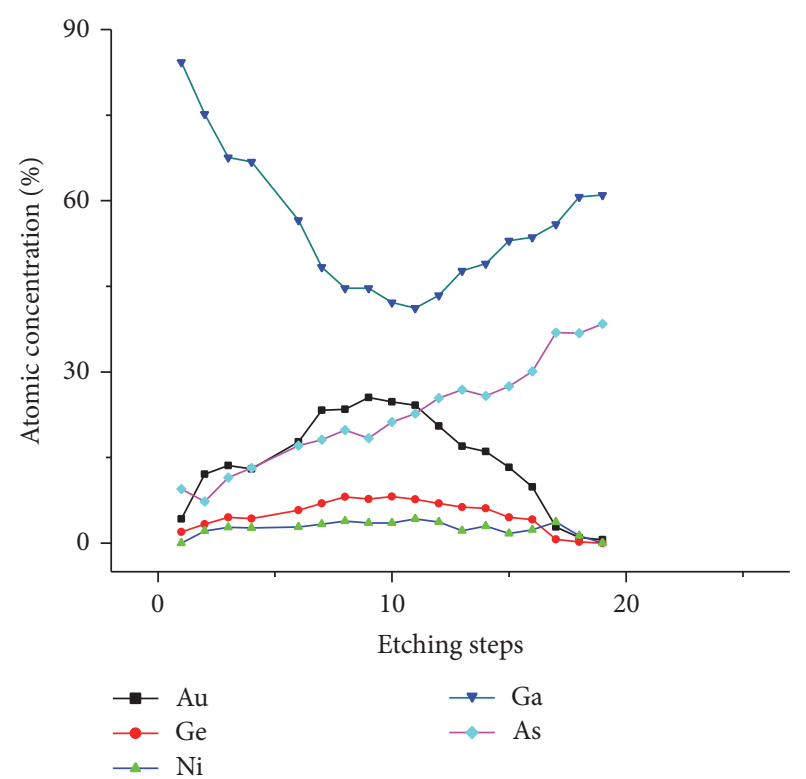

Figure 4: Chemical composition of the ohmic layer.

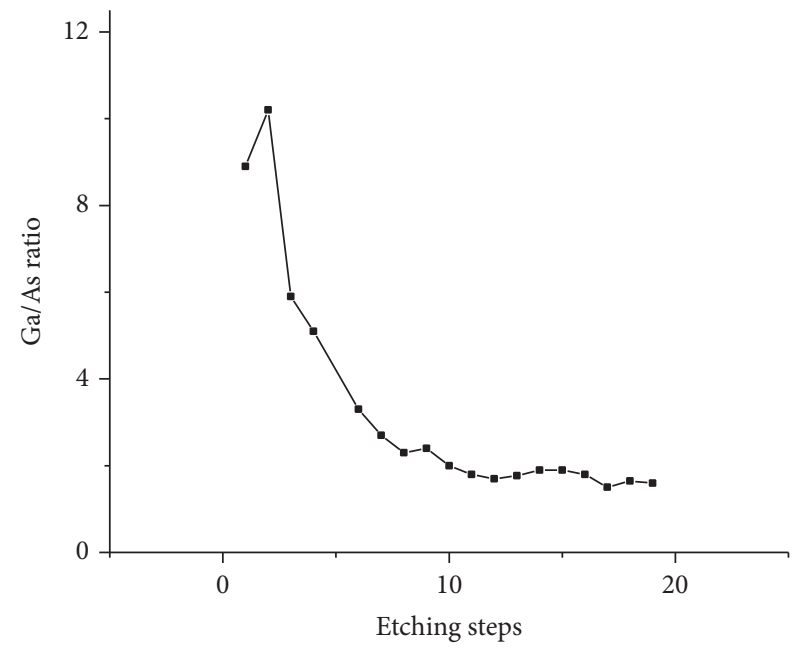

FIGURE 5: Ga/As ratio within the metallic layer.

the surface by ion sputtering. This indicates that both $\mathrm{Ni}$ and Ge diffuse deeper in the $\mathrm{n}$-GaAs semiconductor.

The Ga/As ratio is displayed in Figure 5 and it can be seen that close to the interface and after, this ratio becomes close to 1.5 rather than stoichiometric value of 1 . This has been found in other experiments of GaAs ion etching and is a result of the fact that As is removed preferentially from the surface $[13,14]$. We suppose that another possible loss of As may be due to evaporation during the thermal annealing. Despite the fact that this leads to a higher value of $\mathrm{Ga} / \mathrm{As}$ ratio, the measurements still show an aggressive diffusion of $\mathrm{Ga}$ towards the surface of the metallic layer. Responsible for this are a high value of the diffusion coefficient of $\mathrm{Ga}$ in $\mathrm{Au}$ $\left(10^{-12} \mathrm{~cm}^{2} / \mathrm{sec}\right)$ and the gettering effect of Au over Ga [15].

Both Ga3d and Ga2p spectral lines have been recorded in order to investigate the chemical behavior of $\mathrm{Ga}$. Close 


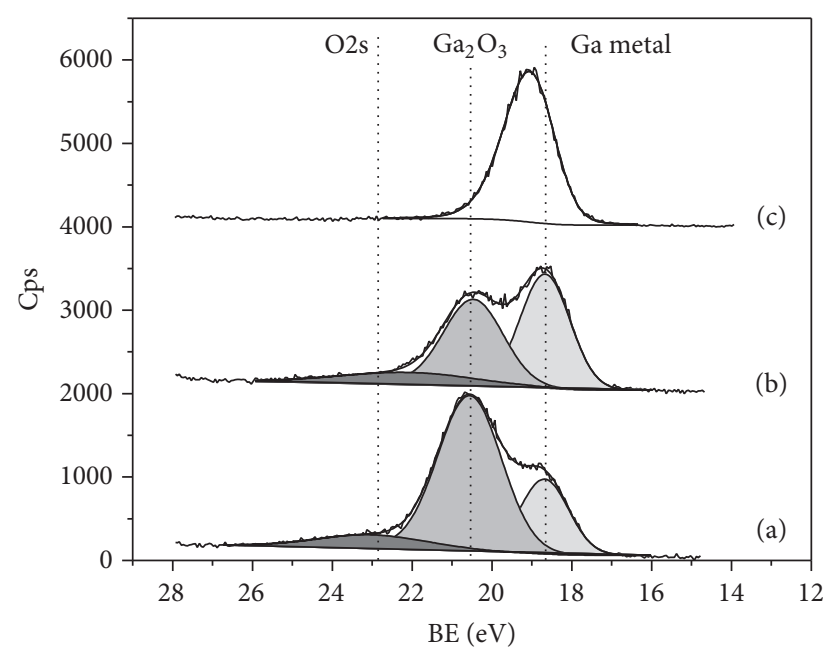

FIGURE 6: XPS spectra of Ga3d spectral line during experiment: (a) before etching, (b) after 3rd etching step, and (c) at the interface.

to Ga3d line there is the O2s spectral line, bringing a bit of difficulty in spectra interpretation. Figure 6 shows the spectra for Ga3d line during measurements. At first $\mathrm{Ga}$ is almost completely oxidized, but after sputtering the surface with $\mathrm{Ar}+$ ions, the metallic component seems to grow and oxide component seems to decrease, until only the metallic state remains visible.

The peaks situated at $20.3 \mathrm{eV}$ binding energy clearly shows the presence of $\mathrm{Ga}_{2} \mathrm{O}_{3}$ while the peaks situated at $18.4 \mathrm{eV}$ are attributed to Ga metal [12]. In the upper layers is improbable the existence of GaAs semiconductor compound, but at the interface and in the last sputtering stages, the binding energy of Ga3d shifts to higher values of 19.1-19.2 eV corresponding to pure GaAs subjected to ion bombardment, as found in the literature [16]. Ga is mainly responsible for fixing the oxygen deep in the layer's structure. Most probably the oxidation of Ga occurs during thermal annealing, and the presence of gallium oxide is observable in the first third of deposited layer's thickness.

As in the case of $\mathrm{Ga}$, both As2p and As3d spectral lines have been recorded. Before sputtering, the As is found in both metallic state and as oxide. The As $2 \mathrm{p}^{3 / 2}$ spectrum indicates only the presence of the $\mathrm{As}_{2} \mathrm{O}_{3}$ compound, while the As3d spectra indicate similar proportions of both metal and oxide. This difference appears because the photoelectrons of As $2 \mathrm{p}^{3 / 2}$ orbital have low kinetic energies and consequently they come from the first two or three atomic layers of the surface, in which the As is fully oxidized. After the first sputtering session, the oxide is no longer visible in the As3d spectra. Close to the interface (Figure 7(b)) the binding energy of the As $3 \mathrm{~d}$ is $40.8 \mathrm{eV}$, corresponding to $\mathrm{GaAs}$ semiconductor whereas in the layer the binding energy is $41.1 \mathrm{eV}$ corresponding to a metallic state or alloy [17]. The As behaves uniformly during the measurements and shows no other state than metallic.

Ge behaves in the same manner as the other metals. Before sputtering, the Ge3d spectrum is composed of two

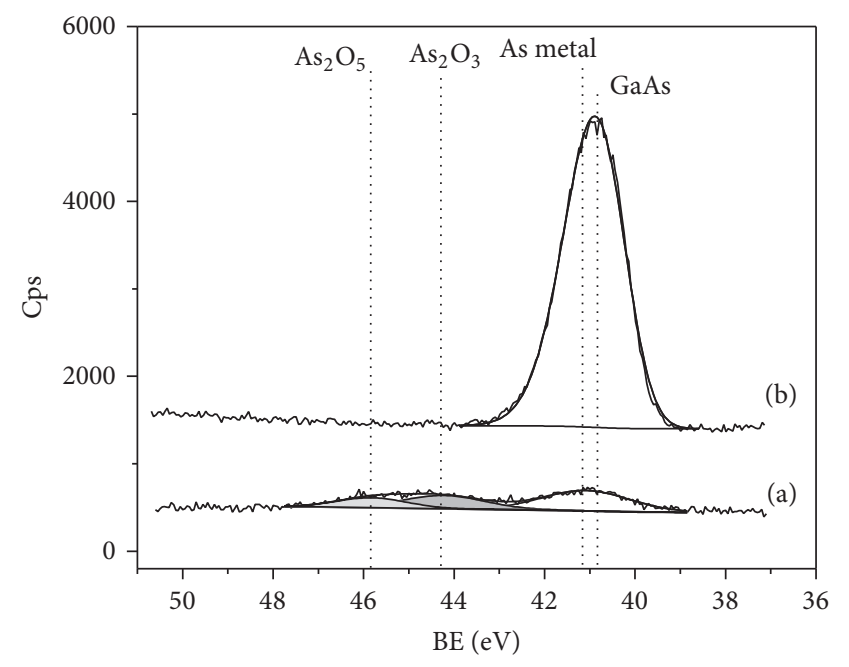

FIgURE 7: XPS spectra of As3d spectral line before etching (a) and at the metal/semiconductor interface (b).

peaks: one situated at $29 \mathrm{eV}$ binding energy and the other at $32.2 \mathrm{eV}$ binding energy.

The first component, representing $32 \%$ from total peak area, is attributed to elemental Ge, while the second component, containing the rest of $68 \%$ from peak area, is undoubtfully attributed to $\mathrm{Ge}_{2} \mathrm{O}_{3}$ specie [18]. Even after the first sputtering session, the oxide completely disappears, with only the Ge metal component remaining. During the measurements, Ge showed a steady stability for both binding energies and FWHM of peak lines (Figure 8). Binding energy ranged between $28.9 \mathrm{eV}$ and $29 \mathrm{eV}$, whereas FWHM was at $1.5 \mathrm{eV} \pm 0.05 \mathrm{eV}$. No other chemical components have been found for Ge. It is widely accepted that the formation of $\mathrm{GeAs}_{2}$ and $\mathrm{Ni}_{3} \mathrm{Ge}$ chemical compounds could inflict higher contact resistance and degradation of ohmic contact parameters. Those two components have binding energies at $29.7 \mathrm{eV}$ and $29.1 \mathrm{eV}$, respectively [19]. However, neither of these components has been identified during the experiment. Attempting to fit the Ge3d spectra with more than one peak provided unsatisfactory results.

$\mathrm{Ni}$ is missing within the first layers of the surface. The $\mathrm{Ni} 2 \mathrm{p}^{3 / 2}$ spectral lines have been recorded. Throughout the experiment the maximum of the $\mathrm{Ni} 2 \mathrm{p}^{3 / 2}$ peak remained stable in a very small energy interval centered around $852.65 \mathrm{eV}$. This binding energy corresponds to a metallic state of $\mathrm{Ni}[20]$. Absence of shake-up satellites indicates once more that there is no Ni oxidized present in the layer. However, close to the interface, the shape of the peak tends to become larger, from a FWHM of $1.6 \mathrm{eV}$ to $1.9 \mathrm{eV}$. This could be explained by the fact that an amount of $\mathrm{Ni}$ interacts directly with GaAs substrate in the semiconductor "wetting" process for which $\mathrm{Ni}$ role is intended. The formation of $\mathrm{Ni}(\mathrm{Ge}) \mathrm{As}$ is also possible, but the chemical shift is too small to be observable by XPS. The presence of Ni even at the interface is beneficial for ohmicity of contacts in that it reduces the lateral diffusion of gold and holds the Au-Ge melt in intimate contact with GaAs substrate during thermal annealing. 


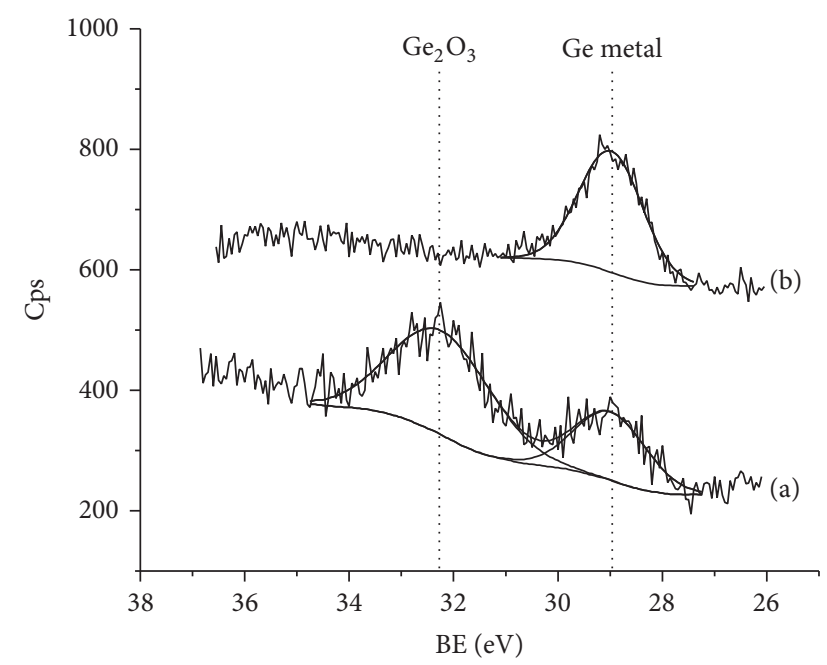

FIGURE 8: XPS spectra of Ge3d spectral line before etching (a) and at the metal/semiconductor interface (b).

For gold, the $4 \mathrm{f}$ doublet has been recorded. The presence of gold is visible from surface to the interface. Figure 9 shows $\mathrm{Au}$ spectrum at the interface. Spectra fitting of $4 \mathrm{f}^{7 / 2}$ line was made with two peaks: one situated at $83.65 \mathrm{eV}$ binding energy and another one having a $84.8 \mathrm{eV}$ binding energy. The first peak is attributed to the metallic gold, while the second is attributed to an alloy of $\mathrm{Ga}$ and $\mathrm{Au}$ [20]. The area of the second peak is less than a quarter of the total area. The Au$\mathrm{Ga}$ alloy was much more investigated previously by high resolution XPS, in various relative proportions of $\mathrm{Ga}$ and $\mathrm{Au}$. The results indicated that the chemical shift of $\mathrm{Au} 4 \mathrm{f}^{7 / 2}$ tends to be growing with the proportion of $\mathrm{Ga}$, from $0.45 \mathrm{eV}$ for $\alpha-\mathrm{Au}_{0.88} \mathrm{Ga}_{0.12}$ to $0.65 \mathrm{eV}$ for $\beta-\mathrm{Au}_{0.78} \mathrm{Ga}_{0.22}, 0.95 \mathrm{eV}$ for $\gamma$ $\mathrm{Au}_{9} \mathrm{Ga}_{4}, 1.25 \mathrm{eV}$ for $\mathrm{Au}-\mathrm{Ga}$, and $1.55 \mathrm{eV}$ for $\mathrm{AuGa}_{2}$ [21]. The chemical shift of $1.15 \mathrm{eV}$ found in our experiment indicates more gold than $\mathrm{Ga}$ in the alloy. The Ga $3 \mathrm{~d}$ and $2 \mathrm{p}$ core levels shifts much less, with a maximum of $0.3 \mathrm{eV}$ for $\mathrm{AuGa}_{2}$, so they are unresolvable in the recorded spectra.

\section{Conclusions}

An experimental procedure consisting in the combination of XPS and in situ Ar+ ion sputtering methods was used as an efficient technique for chemical characterization of $\mathrm{AuGeNi} / \mathrm{n}-\mathrm{GaAs}$ surfaces and interfaces. Information on chemical compounds of the contact layer deposited on nGaAs, diffusion processes, and atomic concentrations of elements has been obtained. The ohmic contact was produced in a new in situ device dedicated for metallic deposition on cleaved semiconductor surfaces.

The depth profile of AuGeNi layer deposited on n-GaAs semiconductor has been evaluated by XPS measurements and by the use of $\mathrm{Ar}+$ ion sputtering technique. Gold, germanium, and nickel are uniformly distributed in the metallic layer. The XPS measurements show that both Ga and As diffuse to the surface, with a more pronounced Ga diffusion. The cleavage technique provides a contaminants-free interface

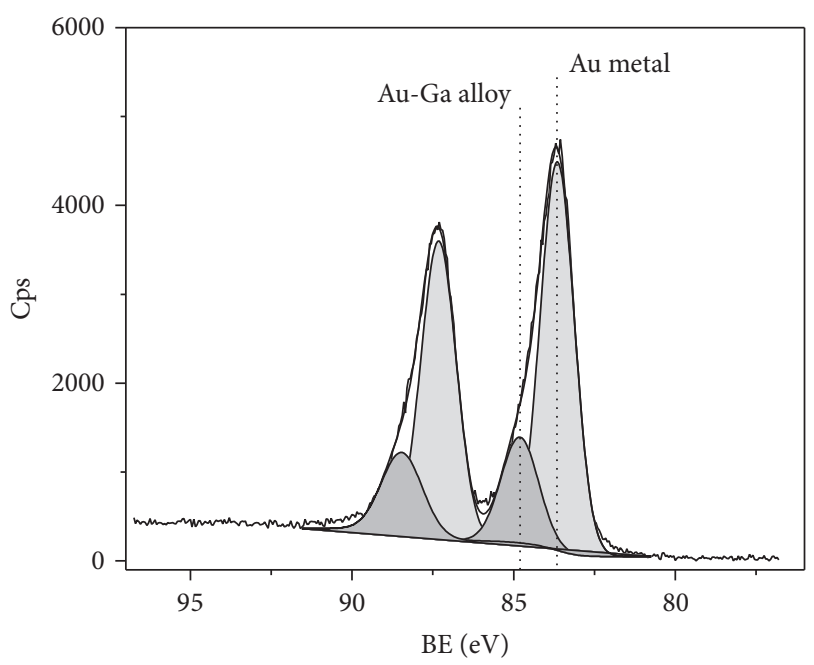

FIGURE 9: XPS spectrum of Au4f spectral line at the metal/semiconductor interface.

(carbon and oxygen). However, there seems to be a quantity of oxygen in the first layers of the sample in the form of gallium oxide. After 20 minutes of Ar sputtering, the oxygen is already absent within the layer. The formation of $\mathrm{Au}$ Ga alloy has been detected, having a good impact over the quality of ohmic contacts. Other components, those who bring negative effects over contacts' ohmicity, like $\mathrm{Ni}_{3} \mathrm{Ge}$ and $\mathrm{GeO}_{2}$, were searched and investigated but not identified in the XPS spectra. Nickel and germanium are both found within the layer and at the interface. They remain in the same proportions at the interface even after the gold concentration begins to decrease. This proves the appropriate diffusion of Ge in the n-GaAs substrate and the wetting of semiconductor surface by $\mathrm{Ni}$.

\section{Disclosure}

The authors confirm that this manuscript has not been published with its full length elsewhere.

\section{Competing Interests}

The authors declare that there is no conflict of interests regarding the publication of this manuscript.

\section{Acknowledgments}

This work was supported by a grant of the Romanian Ministry of Education, CNCS-UEFISCDI, Project no. PN-II-RU-PD2012-3-0051, and the Romanian Research Program "PCCA 2013" Contract no. 68/2014 Partnerships.

\section{References}

[1] M. R. Brozell and C. E. Stillman, Properties of Gallium Arsenide, INSPEC, 1996. 
[2] A. G. Baca, F. Ren, J. C. Zolper, R. D. Briggs, and S. J. Pearton, "A survey of ohmic contacts to III-V compound semiconductors," Thin Solid Films, vol. 308-309, no. 1-4, pp. 599-606, 1997.

[3] M. Murakami, "Development of refractory ohmic contact materials for gallium arsenide compound semiconductors," Science and Technology of Advanced Materials, vol. 3, no. 1, pp. 1-27, 2002.

[4] G. S. Saravanan, K. Mahadeva Bhat, K. Muraleedharan, H. P. Vyas, R. Muralidharan, and A. P. Pathak, "Ohmic contacts to pseudomorphic HEMTs with low contact resistance due to enhanced Ge penetration through AlGaAs layers," Semiconductor Science and Technology, vol. 23, no. 2, Article ID 025019, 2008.

[5] T. Kim and D. D. L. Chung, "In situ X-ray diffraction study of the effects of germanium and nickel concentrations on melting in gold-based contacts to gallium arsenide," Thin Solid Films, vol. 147, no. 2, pp. 177-192, 1987.

[6] T. S. Kuan, P. E. Batson, T. N. Jackson, H. Rupprecht, and E. L. Wilkie, "Electron microscope studies of an alloyed $\mathrm{Au} / \mathrm{Ni} / \mathrm{Au}-$ Ge ohmic contact to GaAs," Journal of Applied Physics, vol. 54, no. 12, pp. 6952-6957, 1983.

[7] P.-Y. Jouan, M.-C. Peignon, Ch. Cardinaud, and G. Lempérière, "Characterisation of TiN coatings and of the TiN/Si interface by X-ray photoelectron spectroscopy and Auger electron spectroscopy," Applied Surface Science, vol. 68, no. 4, pp. 595-603, 1993.

[8] D. Briggs and M. P. Seah, Practical Surface Analyses, vol. 1, John Wiley \& Sons, New York, NY, USA, 1993.

[9] P. A. Zhdan, A. P. Shepelin, Z. G. Osipova, and V. D. Sokolovskii, "The extent of charge localization on oxygen ions and catalytic activity on solid state oxides in allylic oxidation of propylene," Journal of Catalysis, vol. 58, no. 1, pp. 8-14, 1979.

[10] J. A. Taylor, "An XPS study of the oxidation of AlAs thin films grown by MBE," Journal of Vacuum Science and Technology, vol. 20, no. 3, pp. 751-755, 1982.

[11] T. L. Barr, M. Mohsenian, and L. M. Chen, "XPS valence band studies of the bonding chemistry of germanium oxides and related systems," Applied Surface Science, vol. 51, no. 1-2, pp. 7187, 1991.

[12] M. Dürrwächter, G. Indlekofer, H.-G. Boyen, P. Oelhafen, and D. Quitmann, "Core level binding energy shifts in liquid binary alloys: Au-Ga," Journal of Non-Crystalline Solids, vol. 156-158, part 1, pp. 241-245, 1993.

[13] R. V. Ghita, C. Logofatu, C. Negrila et al., "XPS study of Ti/oxidized GaAs interface," Journal of Optoelectronics and Advanced Materials, vol. 8, no. 1, pp. 31-36, 2006.

[14] C. C. Negrila, C. Logofatu, R. V. Ghita et al., "Angle-resolved XPS structural investigation of GaAs surfaces," Journal of Crystal Growth, vol. 310, no. 7-9, pp. 1576-1582, 2008.

[15] P. D. Vyas and B. L. Sharma, "Behaviour of gold in the vicinity of the AuGe nGaAs interface during annealing," Thin Solid Films, vol. 51, no. 2, pp. L21-L23, 1978.

[16] M. Iwami, Y. Watanabe, H. Kato, M. Nakayama, and N. Sano, "Structure of GaAs-In0.2Ga0.8As heterojunction interface studied by electron spectroscopies: X-ray photoelectron spectroscopy, tunable electron energy loss spectroscopy and Auger electron spectroscopy," Thin Solid Films, vol. 146, no. 3, pp. 291-297, 1987.

[17] S. C. Ghosh, M. C. Biesinger, R. R. LaPierre, and P. Kruse, "X-ray photoelectron spectroscopic study of the formation of catalytic gold nanoparticles on ultraviolet-ozone oxidized
GaAs(100)GaAs(100) substrates," Journal of Applied Physics, vol. 101, no. 11, Article ID 114322, 2007.

[18] C. D. Wagner, A. V. Naumkin, A. Kraut-Vass, J. W. Allison, C. J. Powell, and J. R. Rumble Jr., NIST Standard Reference Database 20, Version 3.4, https://srdata.nist.gov/xps/.

[19] G. Hollinger, P. Kumurdjian, J. M. Mackowski, P. Pertosa, L. Porte, and T. M. Duc, "ESCA study of molecular $\mathrm{GeS}_{3-x}$ Tex $\mathrm{As}_{2}$ glasses," Journal of Electron Spectroscopy and Related Phenomena, vol. 5, no. 1, pp. 237-245, 1974.

[20] T. L. Barr, "An ESCA study of the termination of the passivation of elemental metals," Journal of Physical Chemistry, vol. 82, no. 16, pp. 1801-1810, 1978.

[21] D. T. Jayne, N. S. Fatemi, and V. G. Weizer, "Core level x-ray photoelectron spectroscopy of $\mathrm{Au}_{x} \mathrm{Ga}_{y}$ alloys," Journal of Vacuum Science \& Technology A, vol. 10, p. 2802, 1992. 

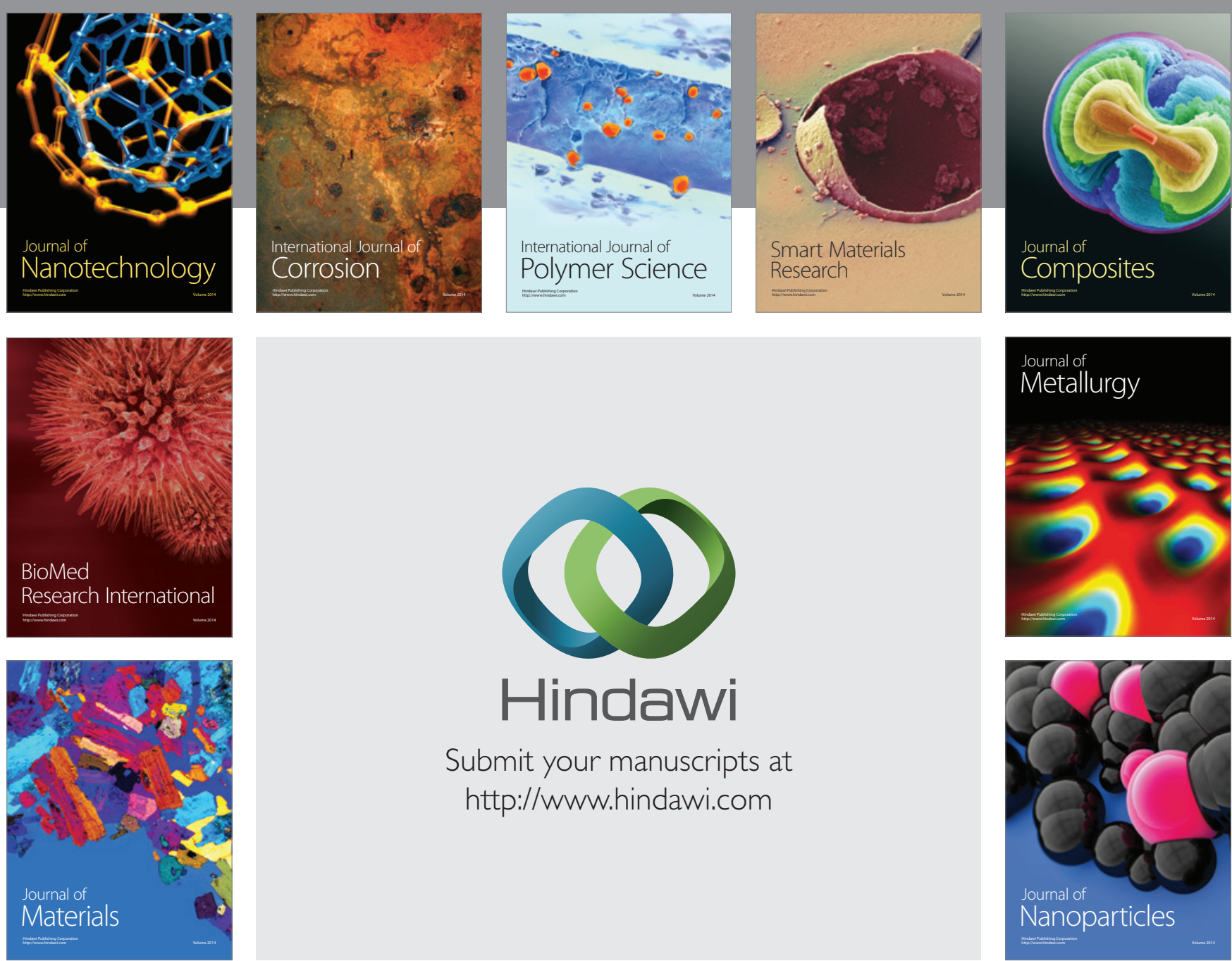

\section{Hindawi}

Submit your manuscripts at

http://www.hindawi.com

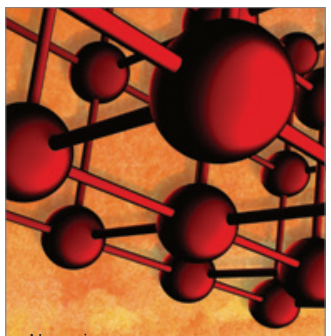

Materials Science and Engineering
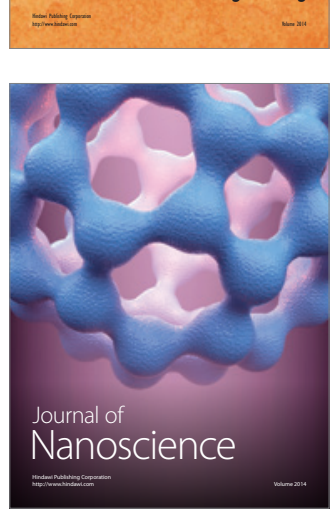
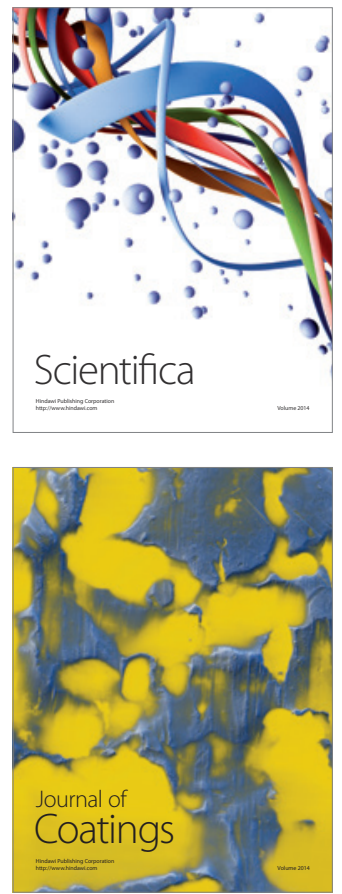
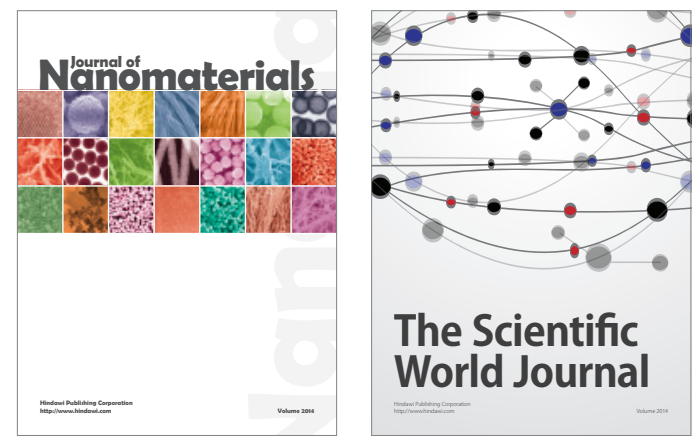

The Scientific World Journal
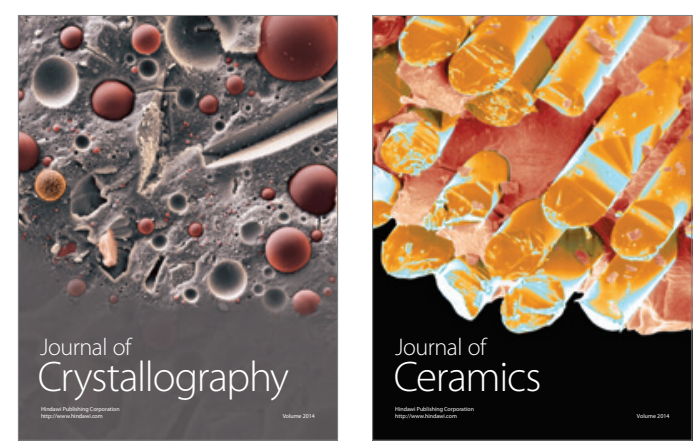
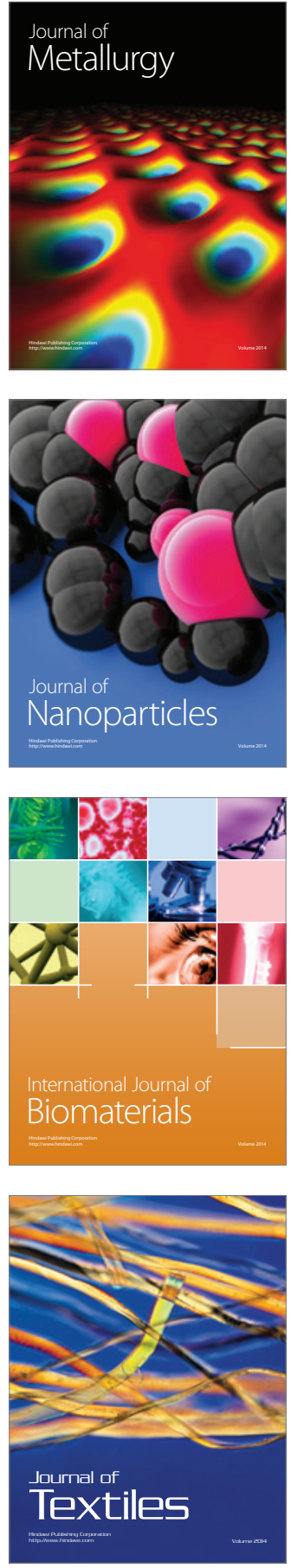